Check for updates

Cite this: RSC Adv., 2019, 9, 12710

Received 7th March 2019

Accepted 3rd April 2019

DOI: 10.1039/c9ra01730e

rsc.li/rsc-advances

\section{A rational design of the coupling mechanism of physical adsorption and chemical charge effect for high-performance lithium-sulfur batteries $\uparrow$}

\author{
Guilin Feng, ${ }^{a}$ Xiaohong Liu, ${ }^{a}$ Yasai Wang, ${ }^{a}$ Zhenguo Wu, (D) ${ }^{a}$ Chen $\mathrm{Wu}^{a}{ }^{a}$ Rong Li, ${ }^{a}$ \\ Yanxiao Chen, (D) ${ }^{* a}$ Xiaodong Guo, ${ }^{a}$ Benhe Zhong ${ }^{a}$ and Jianshu Li $^{\text {b }}$
}

\begin{abstract}
Lithium sulfur batteries are considered as potential energy storage systems for electrical devices owing to their high energy density, low cost, and environmental friendliness. However, the hasty capacity fading originating from the solution and migration of polysulfides is the major obstacle for their industrial application. The polysulfide adsorption and repulsion effect achieved by adding an extra coating layer on the side of the cathode and separator have been separately proved to be effective in mitigating the shuttle effect. Herein, a cooperative coated separator, which employs a hybrid carbon matrix as the coated material, including an appropriate ratio of $\mathrm{N}$-doped activated conductive carbon and commercial acetylene black, and sulfonated polystyrene as the binder, is established to prevent the migration of polysulfides and serves as a secondary current collector to reutilize the active materials for highperformance lithium sulfur batteries. The research results showed that the coated separator with $50 \mathrm{wt} \%$ $\mathrm{N}$-doped activated conductive carbon as the coating material and sulfonated polystyrene as the binder showed highlighted cycle performance, and $731 \mathrm{~mA} \mathrm{~h} \mathrm{~g}^{-1}$ was maintained after 150 cycles at $800 \mathrm{~mA} \mathrm{~g}^{-1}$ (the capacity retention was $86.0 \%$ ). The superior performance may be because the coated separator can efficiently restrain the polysulfides by physical and chemical effects and also reject the polysulfides by the anion electrostatic effect. In summary, this study provides a new cooperative way to address the shuttle effect and promotes the development of lithium sulfur batteries.
\end{abstract}

\section{Introduction}

The rapid growth of electric automobiles and portable electronic devices propels higher performance demands on batteries. Hence, it is extremely urgent to exploit a novel green energy storage lithium-ion battery with high specific energy ${ }^{\mathbf{1 - 4}}$ Among the next alternatives of energy storage systems, lithium sulfur ( $\mathrm{Li}-\mathrm{S})$ batteries are regarded as one of the most potential batteries due to their high theoretical specific capacity (1675 $\mathrm{mA} \mathrm{h} \mathrm{g}^{-1}$ ) and high energy densities (2600 $\mathrm{W} \mathrm{h} \mathrm{kg}^{-1}$ ), which far exceeds those of the traditional lithium-ion batteries. ${ }^{5-9}$ Furthermore, the intrinsic characteristics of the sulfur cathode, such as environment benignity, low cost, and abundant resources, further push forward the progress of $\mathrm{Li}-\mathrm{S}$ batteries. ${ }^{\mathbf{1 0 - 1 3}}$ However, their practical commercial applications are still impeded by the sharp capacity degradation, severe

${ }^{a}$ School of Chemical Engineering, Sichuan University, Chengdu 610065, P. R. China. E-mail: yxchen888@163.com; Fax: +86-28-85406702; Tel: +86-28-85406702

${ }^{b}$ College of Polymer Science and Engineering, State Kay Laboratory of Polymer Materials Engineering, Sichuan University, Chengdu 610065, People's Republic of China

$\dagger$ Electronic supplementary information (ESI) available. See DOI: 10.1039/c9ra01730e volume change, limited coulombic efficiency, transfer of highorder lithium polysulfides (LPSs) into low-order LPSs and the migration of low-order LPSs between the cathode and anode creating the "shuttle effect". ${ }^{14-19}$

To address the abovementioned challenges, significant efforts have been made, and various strategies have been investigated. The general strategy is to anchor sulfur in various carbon materials with fixed special construction and pore sizes. ${ }^{\text {20-22 }}$ Although these strategies could effectively trap LPSs in the cathode and strengthen the electrochemical performance of Li-S batteries, the complex designed structures and the highpriced treatment techniques are inapplicable in large-scale manufacturing processes. Based on the previous studies, a series of functional separators with the electrostatic effect, ${ }^{23,24}$ physical constraint effect, ${ }^{25,26}$ and chemical adsorption effect $^{14,27,28}$ have been designed for simplification. For instance, a particular sulfonated acetylene black $\left(\mathrm{AB}-\mathrm{SO}_{3}{ }^{-}\right)$-coated separator with electronegative coating layers could effectively enhance the performance of $\mathrm{Li}-\mathrm{S}$ batteries, owing to the electrostatic repulsion between the $\mathrm{AB}-\mathrm{SO}_{3}{ }^{-}$and LPSs. ${ }^{29} \mathrm{Zhu}$ et al. ${ }^{30}$ prepared a carbon-based aerogel to modify the separator, which could deliver a reversible discharge capacity, due to the screen size effect of the coated layer. Quan-Hong Yang et al. designed a tight coating composed of $\mathrm{Li}_{4} \mathrm{Ti}_{5} \mathrm{O}_{12}$ and graphene on the 
separator, which could develop an excellent cycle lifetime in $\mathrm{Li}-$ $\mathrm{S}$ batteries $\left(697 \mathrm{~mA} \mathrm{~h} \mathrm{~g}{ }^{-1}\right.$ after 500 cycles at 1C, the capacity retention was $85.7 \%$ ), as a result of the chemical adsorption effect of $\mathrm{Li}_{4} \mathrm{Ti}_{5} \mathrm{O}_{12}$ on LPSs. ${ }^{31}$ Moreover, carbon matrices doped with heteroatoms have been proved to have a large potential for the adsorption of LPSs. ${ }^{32,33}$ Although these modified methods from a single angle have shown improved performances, settling the shuttle effect of $\mathrm{Li}-\mathrm{S}$ batteries still remains a big challenge. Recently, the application of polystyrene sulfonate@HKUST-1 has been demonstrated to increase the capacity, due to the facilitation of lithium ion transport and the electrostatic effect. Besides, the application of $\mathrm{N}, \mathrm{P}$ element in $\mathrm{Li}-\mathrm{S}$ batteries has been further proved its strong chemical adsorption to polysulfides, improving rate and cycling performance. ${ }^{1,34,35}$

Herein, a novel coated separator was devised, as shown in Fig. 1, which employed the sulfonated polystyrene (SPS) as the binder and appropriate ratio of $\mathrm{N}$-doped activated conductive carbon (NACC) and acetylene black (AB) as the coated materials. The coated separator could effectively mitigate the shuttle effect via the repulsive effect and chemical effect of N-doped carbon. Compared with ordinary conductive materials, $\mathrm{N}$-doped carbon could act as a good anchor of LPSs. Moreover, NACC rooted from biomass tea, is low-cost and easily prepared. Besides, SPS has a large repulsive effect due to the functional group $-\mathrm{SO}_{3} \mathrm{H}$. To further explore the effect of SPS-N-doped carbon (S-NACC) in $\mathrm{Li}-\mathrm{S}$ batteries, the AB-coated separator with polyvinylidene fluoride ( $\mathrm{P}-\mathrm{AB})$ as the binder was introduced for comparison; moreover, the S-NACC-coated layer could be used as a screen for the diffusion of LPSs due to the cooperated effect of $-\mathrm{SO}_{3} \mathrm{H}$ and $\mathrm{N}$-doped carbon, which could restrict the soluble LPS species by a physical region and also mitigate the shuttle of soluble LPSs by anion electrostatic effect, leading to a superior rate performance $\left(710 \mathrm{~mA} \mathrm{~h} \mathrm{~g}^{-1}\right.$ at $1600 \mathrm{~mA} \mathrm{~g}^{-1}$ ) and stable cycle stability after 150 cycles (retained $86.0 \%$ at $800 \mathrm{~mA} \mathrm{~g}^{-1}$ ).

\section{Experimental}

\subsection{Synthesizing materials and coated separator}

2.1.1 The synthesis of sulfonated polystyrene (SPS). The diagrammatic drawing of the synthesis of SPS is shown in

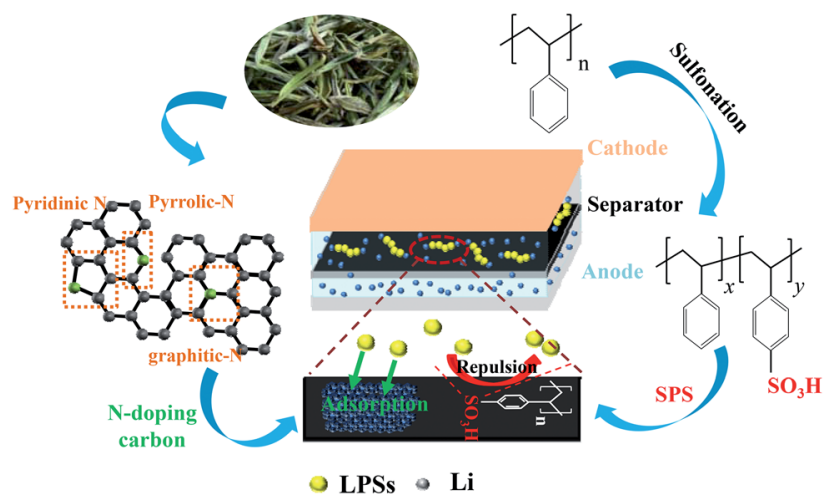

Fig. 1 Working diagrams of S-NACC-coated separator and details of the carbonization of tea and the sulfonation of polystyrene.
Fig. S1.† First, $4.5 \mathrm{~g}$ polystyrene (PS) was dissolved in a threenecked flask with $45 \mathrm{~mL} \mathrm{1,2-dichloroethane} \mathrm{as} \mathrm{the} \mathrm{solvent} \mathrm{at}$ $60{ }^{\circ} \mathrm{C}$. Then, $10 \mathrm{~mL}$ concentrated sulfuric acid (95-98\%) was added into the above solution dropwise. After reacting for 90 minutes under magnetic stirring at $60{ }^{\circ} \mathrm{C}$, the solution was quickly transferred into boiling water to precipitate the prepared SPS for further filtration and collection. Then, the gathered SPS was washed several times with deionized water until the neutral filtrate was obtained, and the final product was dried by freeze drying for 2 days.

2.1.2 Preparing $\mathbf{N}$-doped activated conductive carbon. The $\mathrm{N}$-doped activated conductive carbon (NACC) was prepared by the activated method, according to previous literature. ${ }^{36}$ Ordinary tea was grinded by ball-milling method for two hours, and the powder was pyrolyzed at $350{ }^{\circ} \mathrm{C}$ for $4 \mathrm{~h}$ and at $750{ }^{\circ} \mathrm{C}$ for $8 \mathrm{~h}$ in a horizontal tube furnace with argon to obtain carbonated products. Then the carbonated products were activated by $\mathrm{KOH}$ as follows: a given mass of the products was impregnated using $\mathrm{KOH}$ aqueous solution (the mass ratio of $\mathrm{KOH}$ and products was 4) for $24 \mathrm{~h}$, and then the $\mathrm{KOH}$-products were centrifuged and dried at $80{ }^{\circ} \mathrm{C}$ in an oven for $12 \mathrm{~h}$. Later, the dried mixture was transferred into a container and heated at $800{ }^{\circ} \mathrm{C}$ for $1 \mathrm{~h}$ in a horizontal tube furnace under an argon atmosphere at the heating rate of $5{ }^{\circ} \mathrm{C} \mathrm{min}^{-1}$. After cooling down to room temperature, the activated product was neutralized by $1 \mathrm{M} \mathrm{HCl}$ solution until the $\mathrm{pH}$ was about 7 . Subsequently, the processed product was centrifuged, washed with deionized water, and then dried at $80{ }^{\circ} \mathrm{C}$ in a vacuum oven for $12 \mathrm{~h}$.

2.1.3 The preparation of coated separator. A blade coating method was used to prepare the coated layer. A slurry of the coated material was prepared from a mixture of NACC, AB and SPS in an appropriate weight ratio $(x: y: 10 \%)$ using $N$-methyl$e$-pyrrolidinone (NMP) as the solvent. Then, using an automatic coating machine, the slurry was coated on one side of the pristine separator, and the coated separator was dried at $55{ }^{\circ} \mathrm{C}$ in a vacuum oven for $14 \mathrm{~h}$ after being placed overnight at room temperature. As the control, the AB-P-coated separator with the binder polyvinylidene fluoride (PVDF) was treated by the same process. PVDF Kynar 740, was bought from the Shanghai East Fluorine Chemical Technology Co., Ltd.

\subsection{Preparing sulfur/carbon cathode and assembling cells}

Sulfur was bought from the Chengdu Kelong chemical reagent factory and directly used without other treatments. Ketjen black (KB) powder was purchased from the Shandong Coase new energy co. LTD. The sulfur/carbon (S/C) cathode powder was prepared by mechanical mixing and following the solid state method. The sulfur powder and $\mathrm{KB}$ were mixed at a weight ratio of $4: 1$, and then subjected to ball milling for $40 \mathrm{~min}$. To acquire the experimental $\mathrm{S} / \mathrm{C}$ materials, the mixture was heated at $155{ }^{\circ} \mathrm{C}$ for $12 \mathrm{~h}$ in a tube furnace with Ar atmosphere. And then the heated product as active material (70 $\mathrm{wt} \%$ ) mixed amount of $\mathrm{AB}(20 \mathrm{wt} \%)$ with PVDF (10 wt\%) finally makes up the composite of slurry dispersed in NMP. Then, the slurry was coated on an aluminum foil and dried in a vacuum oven for $12 \mathrm{~h}$ at $80{ }^{\circ} \mathrm{C}$. The electrode with a diameter of $14 \mathrm{~mm}$ was gathered 
by cutting the obtained piece; the average weight of the sulfur$\mathrm{KB}$, acetylene black and PVDF coated on the Al foil was $1.3 \mathrm{mg}$ $\mathrm{cm}^{-2}$.

The batteries were assembled using different separators in a glove box, filled with $\mathrm{Ar}$ atmosphere, and the content of $\mathrm{H}_{2} \mathrm{O}$ and $\mathrm{O}_{2}$ was $0.1 \mathrm{ppm}$. Moreover, $0.65 \mathrm{M}$ lithium bis(trifluoromethanesulfonyl)imide (LiTFSI) solution in 1,2-dimethoxy ethane (DME)/1,3-dioxolane (DOL) $(1: 1, \mathrm{v} / \mathrm{v})$ with $0.1 \mathrm{M}$ $\mathrm{LiNO}_{3}$ additive was used as the electrolyte, and the counter electrode was lithium metal. The addition of the electrolyte was about $30 \mu \mathrm{L}$ in every cell.

\subsection{Characterization}

The surface morphologies of NACC and the separators were detected by means of field emission scanning electron microscopy (SEM, SPA400 Seiko Instruments). Atomic force microscope (AFM) was used to study the surface morphology of the modified separators. Fourier transform infrared spectroscopy (FT-IR) using the $\mathrm{KBr}$ method in the wave number range of 400$4000 \mathrm{~cm}^{-1}$ was carried out on a Nicolet iS50 to observe the $\mathrm{SO}_{3}{ }^{2-}$ of SPS. Thermogravimetric analysis (TGA, TA Instruments, Q600) was used to determine the content of $\mathrm{S}$ with a heat speed of $10{ }^{\circ} \mathrm{C} \min ^{-1}$ from 25 to $500{ }^{\circ} \mathrm{C}$. X-ray photoelectron spectroscopy (XPS) was used to analyze the effect of the coated separator. Raman spectra were gathered with a HORIBA HR800 using $532 \mathrm{~nm}$ laser to observe the property of NACC. The ${ }^{1} \mathrm{H}$ NMR spectra were obtained on an Avance III 400 (Bruker) to determine the DS of the SPS, which employed a solution of the SPS polymer in deuterated dimethyl sulfoxide (DMSO-d6) and tetramethylsilane (TMS) was the internal standard. The energy dispersive X-ray spectrometer (EDX) image was used for morphology analysis. The Brunauer-Emmett-Teller (BET) surface area was obtained from the nitrogen adsorptiondesorption isotherms.

The electrical impedance spectroscopy (EIS) tests of the batteries were conducted using the electrochemical workstation (Germany, ZenniumIM6). The assembled batteries were galvanostatically measured using the LAND-CT2001A battery testing system in the potential window of $1.0-2.8 \mathrm{~V}$ to observe the electrochemical stability of Li-S batteries.

\section{Results and discussion}

The reaction formula of SPS is depicted in Fig. 2a, which details the construction and aromatic proton numbering of SPS. It can be seen that $-\mathrm{H}$ on the counterpoint is likely to be replaced by $-\mathrm{SO}_{3} \mathrm{H}$, because of the electronic effect of polystyrene. The ${ }^{1} \mathrm{H}$ NMR spectra of SPS in DMSO-d6 reflect the ${ }^{1} \mathrm{H}$ NMR spectra of the SPS to confirm the degree of sulfonation (DS $=y /(x+y))$ of SPS. According to the method described in the literature, ${ }^{37}$ the proportional peak area of the distinct peak $\left(2^{\prime}, 2^{\prime}\right)$ at $7.4 \mathrm{ppm}$ denoted as $\left(\mathrm{A}_{2^{\prime}, 2^{\prime}}\right)$ and the integrated peak area of the signals at $7.1 \mathrm{ppm}$ corresponding to all the other aromatic hydrogens denoted as $\left(A_{1^{\prime}}, 1^{\prime}\right)$ are in accord with the following equation:

$$
\frac{2 \mathrm{~d}}{(5-3 \mathrm{~d})}=\frac{\mathrm{A}_{2^{\prime}, 2^{\prime}}}{\mathrm{A}_{1^{\prime}, 1^{\prime}}}(0<d<1)
$$
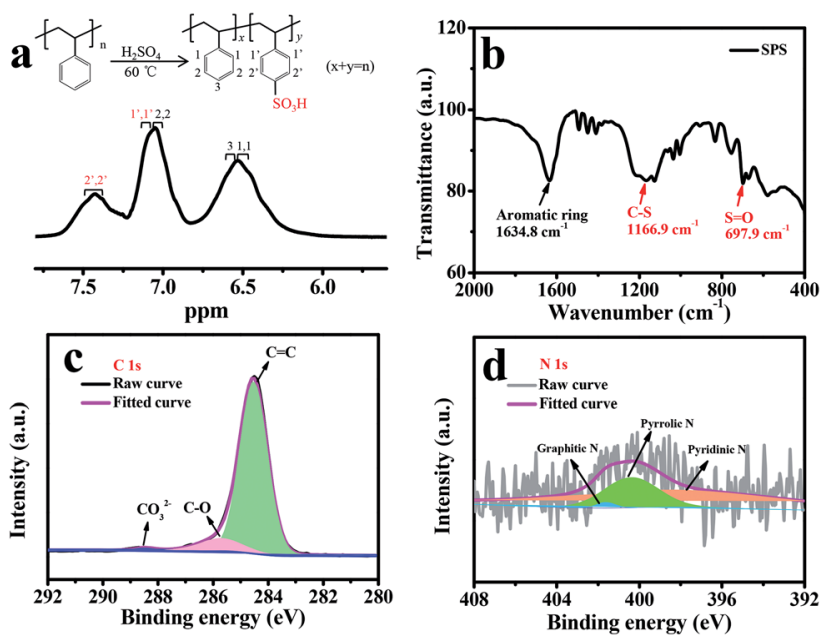

Fig. 2 (a) Reaction formula of SPS and ${ }^{1} \mathrm{H}$ NMR spectra of the prepared SPS in DMSO-d6; (b) the FT-IR spectrum of SPS; (c) XPS survey of $C 1 \mathrm{~s}$ spectra and (d) N 1s spectra for the prepared NACC.

where $d$ is the number of $-\mathrm{SO}_{3} \mathrm{H}$ per repeat unit. The estimated degree of sulfonation is acquired as $\mathrm{DS}=d \times 100 \%$. It could be calculated that the DS of the SPS employed in this research is $76.7 \%$. Fig. $2 \mathrm{~b}$ displays the FT-IR spectrum of SPS, and it can be seen that the stretching vibration of the aromatic ring is at $1634.8 \mathrm{~cm}^{-1}$. The vibrations at 697.9 and $1166.9 \mathrm{~cm}^{-1}$ reflect the stretch vibration of the $\mathrm{C}-\mathrm{S}$ bond and $\mathrm{S}=\mathrm{O}$ bond, respectively, indicating the success of the sulfonation reaction.

The TGA result of the S/C composite powder is shown in Fig. S2. $\dagger$ The sudden weight loss comes from the sublimation of sulfur when the temperature changes to $360^{\circ} \mathrm{C}$, indicating that the sulfur content is about $79.6 \mathrm{wt} \%$, which is in agreement with our designed value (80\%). As shown in Fig. S3, $\uparrow$ NACC displays an irregular morphology after activation. To explore the pore structure and specific surface area, the nitrogen gas adsorptiondesorption isotherms and the distributed pore size of NACC were studied, as displayed in Fig. S4a and b. $\uparrow$ The calculated specific surface area of NACC is $484.2 \mathrm{~m}^{2} \mathrm{~g}^{-1}$ and the cumulative pore diameter is at $3.9 \mathrm{~nm}$, which would be helpful for enhancing the contact area of the electrolyte and providing transport channels for lithium ions. ${ }^{30,38}$ Raman spectroscopy was applied for revealing the structural information and property of the carbon materials, as shown in Fig. S5. $\dagger$ The two peaks at 1336.3 and $1599.1 \mathrm{~cm}^{-1}$ correspond to the D bond and G bond, respectively. The former bond is indexed to the existence of amorphous carbon or deficient graphitized carbon, and the latter band is related to the tangential vibration of the $\mathrm{sp}^{2}$ bonded carbon atoms in the crystallographic graphite structure. ${ }^{34,39}$ In addition, it could be calculated that the peak height ratio of $I_{\mathrm{D}} / I_{\mathrm{G}}$ is 1.01 , which shows a favorable graphitization degree for NACC. The graphitic structure developed in NACC is beneficial for the electronic conductivity.

The surface valances state of $\mathrm{N}$ and $\mathrm{C}$ element in NACC were further explored by XPS. The fitted C 1s spectra (Fig. 2c) of NACC exhibit three peaks at about 284.5, 285.8 and $288.3 \mathrm{eV}$, corresponding to the three categories $\mathrm{C}=\mathrm{C}, \mathrm{C}-\mathrm{O}$ and $\mathrm{CO}_{3}{ }^{2-}, 40,41$ 
respectively. The high content of $\mathrm{C}=\mathrm{C}$ bond is consistent with the results of the Raman spectra. The $\mathrm{N}$ 1s spectra are shown in Fig. 2d, which reveal the presence of the three nitrogen species, graphitic-N (401.2 eV), pyrrolic-N (400.9 eV) and pyridinic-N (398.1 eV), respectively. ${ }^{34,42}$ The three combined ways of $\mathrm{N}$ atom and carbon atom would be helpful for confining the soluble LPSs.

To further study the surface morphology of the modified and initial separators, the atomic force microscope (AFM) technique was applied. Fig. 3a and b shows the 3D topographic AFM images of the different separators; it can be seen that the surface appearance clearly changes after coating, and the coating separator shows a relatively smooth and tight surface. The 2D morphological image of the pristine separator shows a series of pores in micron ranges, but the coated separator (Fig. 3c) shows a close-grained surface, which may be beneficial for blocking LPSs through the separator. The SEM images of the coated separators show three-dimensional porous networks with numerous particle stacking, which could effectively restrict LPSs by a physical region. Nevertheless, the pristine separator has many pores, which are not beneficial to inhibit the migration of LPSs. The result is in keeping with the AFM results. Furthermore, the Raman result of the S-NACC-coated separator is shown in Fig. S5b, $\uparrow$ which is similar with the Raman result of NACC, since the main composites of the coated separator are carbon materials. Fig. $3 \mathrm{~h}$ is the section image of the S-NACC$50 \%$-coated separator; it can be seen that the thicknesses of the Celgard and the coated layer are around 14.1 and $18.3 \mu \mathrm{m}$, respectively.
In order to examine the trapped effect in the coating layer, the SEM and EDX results of the S-NACC-50\%-coated separator are shown in Fig. $3 \mathrm{i}$ and $\mathrm{j}$. It can be seen that there is no obvious sulfur signal in the SEM image before cycling. However, after cycling there is an apparent sulfur signal in the coated layer, which manifests that the S-NACC-50\%-coated separator could effectively restrict the dissolved LPSs by the construction of a barrier region. It's worth mentioning that there is about $0.98 \%$ sulfur in the coated separator before cycling, which could be associated with the addition of SPS $(10 \%)$. This result is in keeping with the ${ }^{1} \mathrm{H}$ NMR test of SPS.

The rate performance of the different separators is depicted in Fig. 4a, and all capacities are on account of the weight of the absolute sulfur material. Among them, from 100 to $1600 \mathrm{~mA} \mathrm{~g}^{-1}$, the S-NACC-50\%-coated separator delivers higher reversible discharge capacities of $\sim 1200$ and $\sim 820 \mathrm{~mA} \mathrm{~h} \mathrm{~g}^{-1}$, respectively, and the discharge capacities of the $\mathrm{P}-\mathrm{AB}$-coated separator are lower, $\sim 1000$ and $\sim 710 \mathrm{~mA} \mathrm{~h} \mathrm{~g}{ }^{-1}$, respectively. In contrast, the conventional separator only maintains the capacities of $\sim 700$ and $\sim 490 \mathrm{~mA} \mathrm{~h} \mathrm{~g}{ }^{-1}$, respectively. The rate capabilities of the three samples obviously indicate that the $\mathrm{S}$ NACC-50\%-coated separator is better than the P-AB-coated separator and the conventional one in $\mathrm{Li}-\mathrm{S}$ batteries. What's more, $960 \mathrm{~mA} \mathrm{~h} \mathrm{~g}{ }^{-1}$ could be maintained when the current density changed to $200 \mathrm{~mA} \mathrm{~g}^{-1}$ because the NACC-50\%-coated separator could effectively mitigate the shuttle effect of LPSs in Li-S batteries. The initial charge-discharge curves of the different separators at $800 \mathrm{~mA} \mathrm{~g}^{-1}$ are shown in Fig. $4 \mathrm{~b}$. The voltage curves of all the batteries express a similar voltage
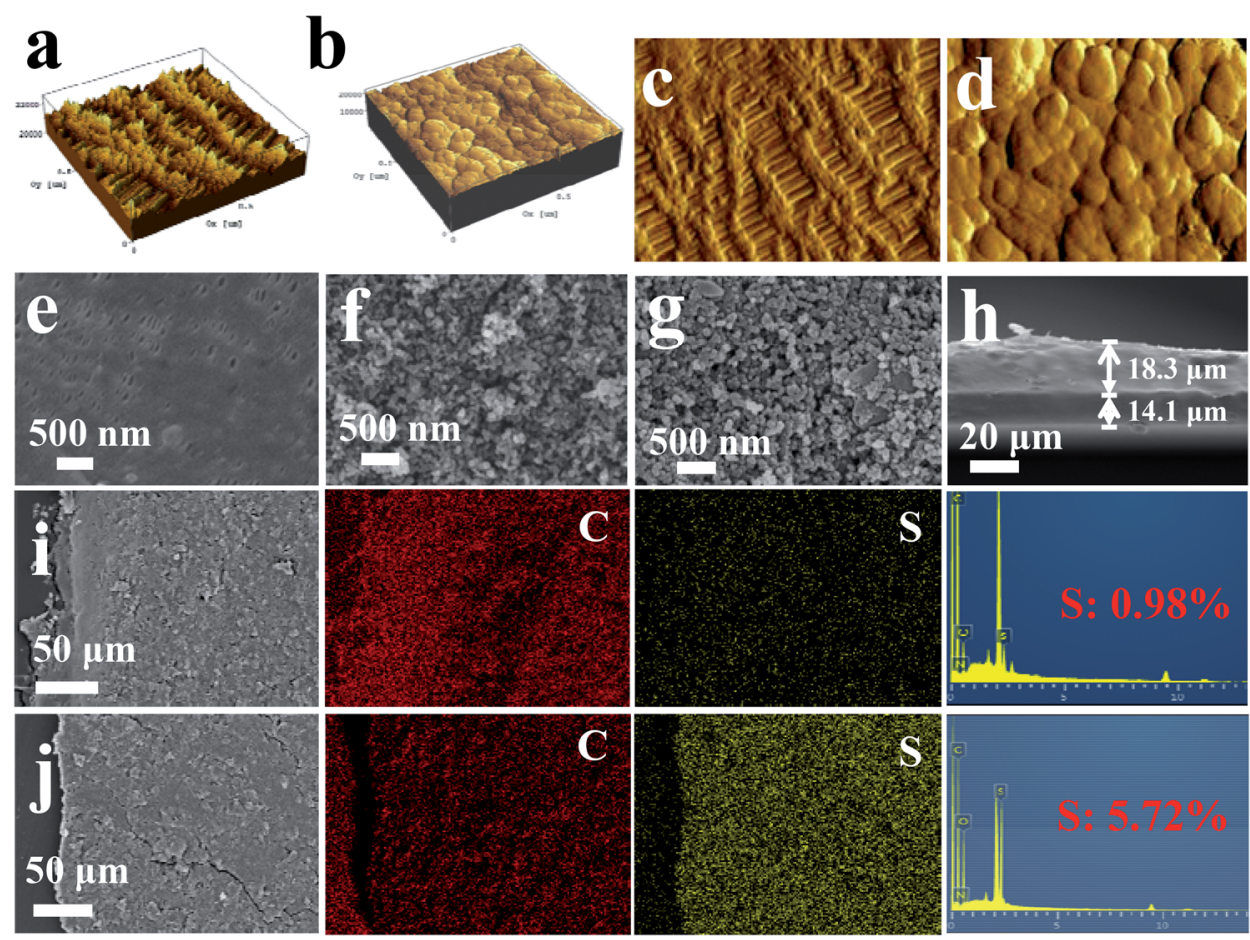

Fig. 3 AFM 3D morphological images of (a) pristine and (b) NACC-coated separator; 2D morphological images of (c) routine separator and (d) coated separator; SEM images of (e) routine separator, (f) P-AB-coated separator and (g) S-NACC-50\%-coated separator; (h) thickness of SNACC-50\%-coated separator; (i) the EDS elemental maps of S-NACC-50\% before cycling, (j) after cycling. 

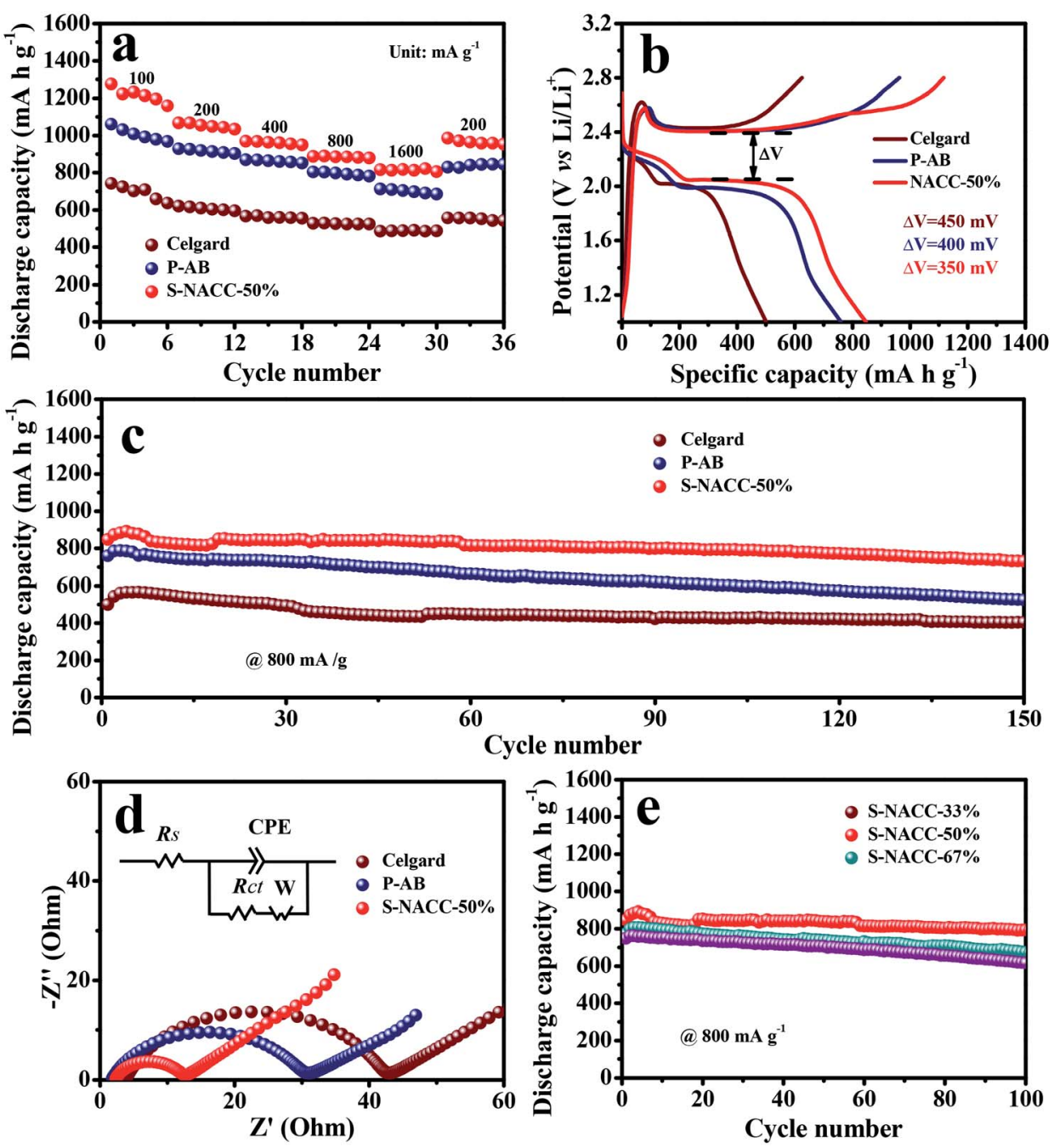

Fig. 4 (a) Rate capacity, (b) the three different charging-discharging curves at $800 \mathrm{~mA} \mathrm{~g}^{-1}$, (c) cycling performance at $800 \mathrm{~mA} \mathrm{~g}{ }^{-1}$, and (d) EIS of the three samples without any measurement and schematic of equivalent circuit (inset); (e) cycling performance at the current density of $800 \mathrm{~mA} \mathrm{~g}^{-1}$ for different additions of NACC.

platform during the discharge-charge processes, which consists of two discharging plateaus, a short higher plateau at $2.30 \mathrm{~V}$ and a long lower plateau at $2.10 \mathrm{~V}$. Additionally, only a long plateau during the charging process. The two plateaus during the discharging-charging processes correspond to the two-step sulfur redox reactions. Specially, the former plateau at $2.30 \mathrm{~V}$ shows that the sulfur obtained electrons and yielded long-chain LPSs $\left(\mathrm{Li}_{2} \mathrm{~S}_{x}, 4<x<8\right)$, and the latter plateau at $2.10 \mathrm{~V}$ is associated with the further transformation of the long-chain LPSs into lower-order $\mathrm{Li}_{2} \mathrm{~S}_{2}$ and $\mathrm{Li}_{2} \mathrm{~S}$. The voltage gaps between the charge and discharge plateaus of the pristine sample, P-AB sample and S-NACC-50\% sample are gradually decreasing, indicating that the S-NACC-50\% sample possesses the lowest polarization and better dynamitic performance. The corresponding $\mathrm{d} Q / \mathrm{d} V$ results of the three different separators are shown in Fig. S6, $\dagger$ which are in agreement with the results of the rate capacities.

Fig. $4 \mathrm{c}$ presents the cycling performance of the three samples at $800 \mathrm{~mA} \mathrm{~g}^{-1}$. The initial discharge capacities of the S-NACC-
$50 \%, \mathrm{P}-\mathrm{AB}$ and conventional separator are 847.5, 760.3 and $543.4 \mathrm{~mA} \mathrm{~h} \mathrm{~g}^{-1}$, respectively. In addition, the S-NACC-50\% sample maintains a capacity of $731.3 \mathrm{~mA} \mathrm{~h} \mathrm{~g}^{-1}$ after 150 cycles, and the capacity retention is $86.0 \%$. Nevertheless, the capacity of the $\mathrm{P}-\mathrm{AB}$ sample is $529.9 \mathrm{~mA} \mathrm{~h} \mathrm{~g}{ }^{-1}$, corresponding to a capacity retention of $69.7 \%$. The capacity of the Celgard sample is $404.7 \mathrm{~mA} \mathrm{~h} \mathrm{~g}^{-1}$ (the capacity retention is $74.4 \%$ ). It is noticeable that the Li-S cells using the S-NACC-50\%-modified separator deliver a more stable cycling performance than the PAB-coated and pristine Celgard; the low capacity attenuation may be attributed to the LPSs prevented by the repulsive effect and the effect of the functional surface groups. The EIS measurement results of fresh batteries with the three separators are shown in Fig. 4d. It is clearly seen that the three samples display the representative semicircles at high frequencies and a short oblique line in the low-frequency area. The highfrequency semicircle represents the charge-transfer resistance $\left(R_{\mathrm{ct}}\right)$ of the sulfur cathode, and the low-frequency sloping lines reflect the lithium-ion diffusion resistance within the 

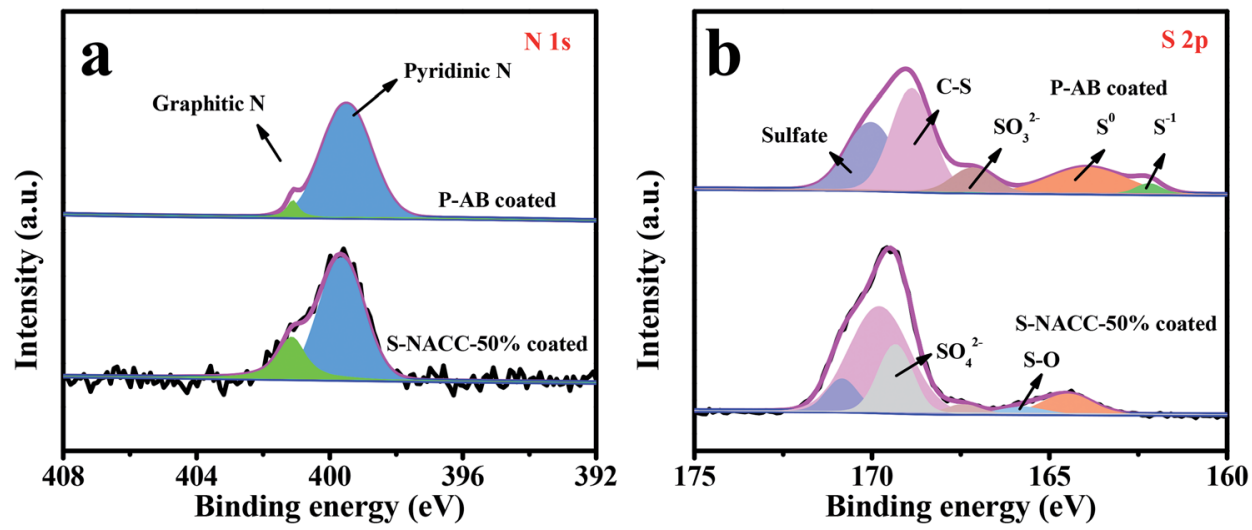

Fig. 5 XPS spectra of (a) N 1s, and (b) S 2p for P-AB-coated separator and S-NACC-50\%-coated separator after cycling.

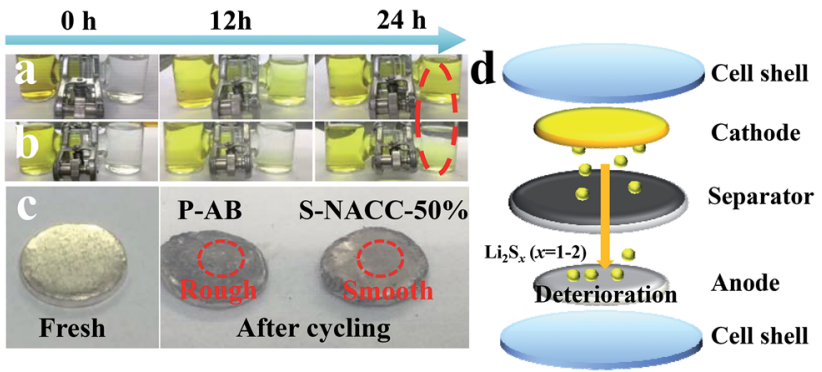

Fig. 6 (a) The Polysulfide rejection tests for P-AB-coated separator and (b) S-NACC-50\%-coated separator. (c) The surface of Li anode before and after cycling. (d) The diagram of $\mathrm{Li}_{2} \mathrm{~S}_{x}(x=1,2)$ deteriorating the surface of lithium.

electrodes. ${ }^{43}$ From correlative equivalent circuit models, the cell with the S-NACC-50\%-coated separator showed a $R_{\mathrm{ct}}$ value of 9.9 $\Omega$, which is lower than that of the cell with the P-AB-coated separator $(26.4 \Omega)$ and conventional separator $(38.0 \Omega)$. It could be guessed that the charge-transfer resistance would be reduced by the addition of SPS and NACC, which could decrease the kinetics impedance, and the result is corresponding to the cycle performance tests.

The self-discharge behaviors are mainly impacted by the shuttle effect in Li-S batteries, which has a close connection with the industrial application. ${ }^{44}$ To evaluate the performance of the three samples, the open circuit potential of $\mathrm{Li}-\mathrm{S}$ batteries was recorded with the change of time, which is shown in Fig. S7. $\dagger$ The open voltage of $\mathrm{Li}-\mathrm{S}$ batteries with the pristine separator rapidly decayed to $2.30 \mathrm{~V}$ while the open voltage of those with the P-AB- and S-NACC-50\%-coated separators were about $2.32 \mathrm{~V}$, within $6 \mathrm{~h}$. This indicted that high-order LPSs turned into low-order LPSs during the self-discharging measurement. As for the coated separator, the capacity retention is higher than that for an uncoated separator.

To better understand the effect of the addition of NACC, different ratios of NACC additions in the coated separator were compared. The cells with S-NACC-33\% (the mass ratio of NACC and $\mathrm{AB}$ is $1: 2$ ), S-NACC-50\% and S-NACC-67\% (the mass ratio of NACC and AB is $2: 1$ ) were studied. Research shows that the additions of NACC have an impact on the performance of $\mathrm{Li}-\mathrm{S}$ batteries. Fig. S8 $\dagger$ shows the rate performance of the three samples. It could be seen that the S-NACC-50\% presented a better performance than the S-NACC-33\% and S-NACC-67\% at various rates, especially at $1600 \mathrm{~mA} \mathrm{~g}^{-1}$. Furthermore, Fig. 4e presents the cycling performances of the three samples at $800 \mathrm{~mA} \mathrm{~g}^{-1}$. The initial discharge capacities are 847, 775 and $749 \mathrm{~mA} \mathrm{~h} \mathrm{~g}{ }^{-1}$, respectively. After 100 cycles, the S-NACC-50\% delivers a capacity of $794 \mathrm{~mA} \mathrm{~h} \mathrm{~g}^{-1}$ and the capacity retention is $93.7 \%$. The rate and cycle performances for different ratios of NACC additions demonstrate that S-NACC-50\% may be more beneficial to remit the shuttle effect; the chemical and physical effect are influenced by the proper ratio used. Because of the irregular morphology, NACC may not be beneficial to trap LPSs, but the character of including $\mathrm{N}$ would show good chemical effect on LPSs. So the use of a proper ratio of NACC in the coated separator is feasible. To further research the cycle performance of S-NACC-50\%, the cycle performance at $1600 \mathrm{~mA} \mathrm{~g}^{-1}$ after 100 cycles was studied, as presented in Fig. S9; $\dagger$ the acquired capacity was $620 \mathrm{~mA} \mathrm{~h} \mathrm{~g}{ }^{-1}$, corresponding to a capacity retention of $88.1 \%$.

To further explore the function mechanism of the S-NACC$50 \%$-coated separator on LPSs, the XPS spectra of P-AB- and SNACC-50\%-coated separator were obtained, as shown in Fig. 5. It can be seen that there are mainly two $\mathrm{N}$ species, graphitic- $\mathrm{N}$ (401.1 eV) and pyridinic-N (398.4 eV), in Fig. 5a. ${ }^{34,45}$ Particularly, the content of graphitic-N in the S-NACC-50\% sample is higher that in the $\mathrm{P}-\mathrm{AB}$ sample, which may be due to the introduction of NACC which innately consists of natural nitrogen, and the results are in keeping with the XPS analysis of NACC. As shown in Fig. 5b, for the two samples, the peaks of $S^{0}$ (164.2 eV), S-O (165.7 eV), $\mathrm{SO}_{3}{ }^{2-}(167.5 \mathrm{eV}), \mathrm{C}-\mathrm{S}(169.5 \mathrm{eV})$ and sulfate $(170.3 \mathrm{eV})^{\mathbf{4 6}}$ are detected; the detected sulfur signals after cycling indicate that the active materials are effectively trapped in the coated separator. Paying attention to the P-AB-coated separator, the peak of $\mathrm{S}^{-1}(162.6 \mathrm{eV})$ shows a discharge product of $S$ cathode. However, the peak of $S^{-1}$ couldn't be detected in the S-NACC-50\%-coated separator and the content of $S^{0}$ is lesser than that in the P-AB-coated separator, which may be associated with the repulsive effect of SPS, resulting in less 
LPSs being deposited on the coated separator. This result demonstrates the strong repulsive effect of SPS for LPSs.

In order to visually verify the effect of the two types of coated separators, polysulfide diffusion experiments were conducted, as shown in Fig. 6a and b. According to previous studies, ${ }^{47}$ the polysulfide $\left(\mathrm{Li}_{2} \mathrm{~S}_{6}\right)$ solution was prepared for this experiment. Comparing the change of color after $24 \mathrm{~h}$, it could be seen that the S-NACC-50\%-coated separator shows a better rejection to polysulfides than the P-AB-coated separator. This implies that the S-NACC-50\%-coated separator could effectively reject the LPSs and mitigate the shuttle effect. Fig. 6c presents the surfaces of Li anode; ${ }^{48}$ it can be seen that the surface is smooth for fresh $\mathrm{Li}$ anode. However, the surfaces have changed after cycling. For the P-AB-coated separator, the surface is rougher than for the S-NACC-50\%-coated separator, indicating that more LPSs move to the $\mathrm{Li}$ anode and react with $\mathrm{Li}$ anode; the process of deterioration is shown in Fig. $6 \mathrm{~d}$.

\section{Conclusion}

In summary, a S-NACC-50\%-coated separator was designed by a combination of the physical effect, chemical effect and repulsive barrier to mitigate the shuttle effect of LPSs in Li-S batteries. The enhancement of $\mathrm{Li}-\mathrm{S}$ batteries could be strongly related with the application of NACC and SPS. For one thing, the conductive carbon (NACC and AB)-coated separator could trap LPSs by the physical and chemical effect and reutilize the trapped LPSs as the upper current collector. For another, the introduction of SPS could reject the LPSs by the repulsive effect, due to the existence of $-\mathrm{SO}_{3} \mathrm{H}$ in SPS. The S-NACC-50\%-coated separator could obtain a discharge capacity of $731 \mathrm{~mA} \mathrm{~h} \mathrm{~g}$ after 150 cycles at $800 \mathrm{~mA} \mathrm{~g}^{-1}$. Hence, the design of the S-NACC$50 \%$-coated separator could be regarded as an effective route to improve the electrochemical performance of $\mathrm{Li}-\mathrm{S}$ batteries and promote their practical application.

\section{Conflicts of interest}

There are no conflicts of interest.

\section{Acknowledgements}

This work was financially supported by the National Natural Science funds (21534008).

\section{References}

1 Y. Guo, M. Sun, H. Liang, W. Ying, X. Zeng, Y. Ying, S. Zhou, C. Liang, Z. Lin and X. Peng, ACS Appl. Mater. Interfaces, 2018, 10, 30451-30459.

2 W. Chen, T. Lei, W. Lv, Y. Hu, Y. Yan, Y. Jiao, W. He, Z. Li, C. Yan and J. Xiong, Adv. Mater., 2018, 1804084.

3 R. Fang, K. Chen, L. Yin, Z. Sun, F. Li and H. M. Cheng, Adv. Mater., 2018, 1800863.

4 Z. Gao, Y. Schwab, Y. Zhang, N. Song and X. Li, Adv. Funct. Mater., 2018, 28, 1800563.
5 X. Liu, T. Qian, J. Liu, J. Tian, L. Zhang and C. Yan, Small, 2018, 14, 1801536.

6 Q. Liu, J. Zhang, S. A. He, R. Zou, C. Xu, Z. Cui, X. Huang, G. Guan, W. Zhang, K. Xu and J. Hu, Small, 2018, 14, 1703816.

7 Y. Zheng, H. Fan, H. Li, C. Fan, H. Yuan, Z. Yang, K. Huang, W. Li and J. Zhang, Chemistry, 2018, 24, 13582-13588.

8 J. He, Y. Chen, W. Lv, K. Wen, C. Xu, W. Zhang, Y. Li, W. Qin and W. He, ACS Nano, 2016, 10, 10981-10987.

9 J. He, Y. Chen, W. Lv, K. Wen, P. Li, F. Qi, Z. Wang, W. Zhang, Y. Li, W. Qin and W. He, J. Power Sources, 2016, 327, 474-480.

10 Y. He, S. Bai, Z. Chang, Q. Li, Y. Qiao and H. Zhou, J. Mater. Chem. A, 2018, 6, 9032-9040.

11 G. Feng, X. Liu, Y. Liu, Z. Wu, Y. Chen, X. Guo, B. Zhong, W. Xiang and J. Li, Electrochim. Acta, 2018, 283, 894-903.

12 W. Chen, T. Lei, T. Qian, W. Lv, W. He, C. Wu, X. Liu, J. Liu, B. Chen, C. Yan and J. Xiong, Adv. Energy Mater., 2018, 1702889, DOI: 10.1002/aenm.201702889.

13 T. Lei, W. Chen, W. Lv, J. Huang, J. Zhu, J. Chu, C. Yan, C. Wu, Y. Yan, W. He, J. Xiong, Y. Li, C. Yan, J. B. Goodenough and X. Duan, Joule, 2018, 2, 2091-2104.

14 L. Hu, C. Dai, H. Liu, Y. Li, B. Shen, Y. Chen, S.-J. Bao and M. Xu, Adv. Energy Mater., 2018, 8, 1800709.

15 X. Chen, L. Yuan, Z. Hao, X. Liu, J. Xiang, Z. Zhang, Y. Huang and J. Xie, ACS Appl. Mater. Interfaces, 2018, 10, 13406-13412.

16 J. He, Y. Chen, W. Lv, K. Wen, C. Xu, W. Zhang, W. Qin and W. He, ACS Energy Lett., 2016, 1, 820-826.

17 W. Chen, T. Lei, W. Lv, Y. Hu, Y. Yan, Y. Jiao, W. He, Z. Li, C. Yan and J. Xiong, Adv. Mater., 2018, e1804084, DOI: 10.1002/adma.201804084.

18 W. Chen, T. Lei, C. Wu, M. Deng, C. Gong, K. Hu, Y. Ma, L. Dai, W. Lv, W. He, X. Liu, J. Xiong and C. Yan, Adv. Energy Mater., 2018, 8, 1702348.

19 W. Chen, T. Lei, T. Qian, W. Lv, W. He, C. Wu, X. Liu, J. Liu, B. Chen, C. Yan and J. Xiong, Adv. Energy Mater., 2018, 1702889, DOI: 10.1002/aenm.201702889.

20 T. Ma, M. Liu, T. Huang and A. Yu, J. Power Sources, 2018, 398, 75-82.

21 H. Zhao, N. Deng, J. Yan, W. Kang, J. Ju, Y. Ruan, X. Wang, X. Zhuang, Q. Li and B. Cheng, Chem. Eng. J., 2018, 347, 343-365.

22 Y. Wei, Z. Kong, Y. Pan, Y. Cao, D. Long, J. Wang, W. Qiao and L. Ling, J. Mater. Chem. A, 2018, 6, 5899-5909.

23 D. B. Babu, K. Giribabu and K. Ramesha, ACS Appl. Mater. Interfaces, 2018, 10, 19721-19729.

24 H. Zhang, C. Lin, X. Hu, B. Zhu and D. Yu, ACS Appl. Mater. Interfaces, 2018, 10, 12708-12715.

25 M. Li, C. Wang, L. Miao, J. Xiang, T. Wang, K. Yuan, J. Chen and Y. Huang, J. Mater. Chem. A, 2018, 6, 5862-5869.

26 Q. Zhao, Q. Zhu, J. Miao, Z. Guan, H. Liu, R. Chen, Y. An, F. $\mathrm{Wu}$ and B. Xu, ACS Appl. Mater. Interfaces, 2018, 10, 10882-10889.

27 J. Wang, J. Liang, J. Wu, C. Xuan, Z. Wu, X. Guo, C. Lai, Y. Zhu and D. Wang, J. Mater. Chem. A, 2018, 6, 6503-6509.

28 P. J. Han, S. H. Chung and A. Manthiram, ACS Appl. Mater. Interfaces, 2018, 10, 23122-23130. 
29 F. L. Zeng, Z. Q. Jin, K. G. Yuan, S. Liu, X. Cheng, A. B. Wang, W. K. Wang and Y. S. Yang, J. Mater. Chem. A, 2016, 4, 1231912327.

30 L. Zhu, L. You, P. Zhu, X. Shen, L. Yang and K. Xiao, ACS Sustainable Chem. Eng., 2017, 6, 248-257.

31 Y. Zhao, M. Liu, W. Lv, Y.-B. He, C. Wang, Q. Yun, B. Li, F. Kang and Q.-H. Yang, Nano Energy, 2016, 30, 1-8.

32 G. Ma, Z. Wen, Q. Wang, C. Shen, P. Peng, J. Jin and X. Wu, J. Power Sources, 2015, 273, 511-516.

33 S. A. Abbas, J. Ding, S. H. Wu, J. Fang, K. M. Boopathi, A. Mohapatra, L. W. Lee, P. C. Wang, C. C. Chang and C. W. Chu, ACS Nano, 2017, 11, 12436-12445.

34 P. Zeng, L. Huang, X. Zhang, R. Zhang, L. Wu and Y. Chen, Chem. Eng. J., 2018, 349, 327-337.

35 T. Lei, W. Chen, Y. Hu, W. Lv, X. Lv, Y. Yan, J. Huang, Y. Jiao, J. Chu, C. Yan, C. Wu, Q. Li, W. He and J. Xiong, Adv. Energy Mater., 2018, 8, 1802441.

36 C. Peng, X.-b. Yan, R.-t. Wang, J.-w. Lang, Y.-j. Ou and Q.-j. Xue, Electrochim. Acta, 2013, 87, 401-408.

37 Q. Xie, Y. Li, X. Chen, J. Hu, L. Li and H. Li, J. Power Sources, 2015, 282, 489-497.

38 Z. Chang, B. Ding, H. Dou, J. Wang, G. Xu and X. Zhang, Chemistry, 2018, 24, 3768-3775.
39 Y. Xie, L. Fang, H. Cheng, C. Hu, H. Zhao, J. Xu, J. Fang, X. Lu and J. Zhang, J. Mater. Chem. A, 2016, 4, 15612-15620.

40 W. Fan, N.-W. Li, X. Zhang, S. Zhao, R. Cao, Y. Yin, Y. Xing, J. Wang, Y.-G. Guo and C. Li, Adv. Sci., 2018, 5, 1800559.

41 F. Qian, J. Shao, Y. Chen, G. Zhu, Q. Qu and H. Zheng, Electrochem. Energy Technol., 2018, 4, 39-46.

42 L. Yao, X. Dong, C. Zhang, N. Hu and Y. Zhang, J. Mater. Chem. A, 2018, 6, 11260-11269.

43 X. Huang, B. Luo, R. Knibbe, H. Hu, M. Lyu, M. Xiao, D. Sun, S. Wang and L. Wang, Chemistry, 2018, 24, 18544-18550.

44 S. Suriyakumar, A. M. Stephan, N. Angulakshmi, M. H. Hassan and M. H. Alkordi, J. Mater. Chem. A, 2018, 6, 14623-14632.

45 J. Zhu, Y. Li, S. Kang, X.-L. Wei and P. K. Shen, J. Mater. Chem. A, 2014, 2, 3142.

46 L. Lodovico, A. Varzi and S. Passerini, J. Electrochem. Soc., 2017, 164, A1812-A1819.

47 P. J. Kim, H. D. Fontecha, K. Kim and V. G. Pol, ACS Appl. Mater. Interfaces, 2018, 10, 14827-14834.

48 J. Zhu, Y. Ge, D. Kim, Y. Lu, C. Chen, M. Jiang and X. Zhang, Nano Energy, 2016, 20, 176-184. 